\title{
2-Nonadecanone Alleviates Depression through Inflammation Relief in SD Rat
}

\author{
Gil-Hyun Lee ${ }^{1, *}$ and Kyung-Yae Hyun ${ }^{2, \dagger ; *}$ \\ ${ }^{1}$ Department of Clinical Laboratory Science, Kyungwon University, Gumi Gyungbuk 39160, Korea \\ ${ }^{2}$ Department of Clinical Laboratory Science, Dong-Eui University, Busan 47340, Korea
}

\begin{abstract}
Depression is a type of mood disorder characterized by hypochondriasis, decreased appetite, and insomnia. Depression is a disease that affects more than 100 million people worldwide. 2-Nonadecanone (NAC) is a bioactive substance that constitutes Fomes fomentarius, and NAC is expected to have an antidepressant effect. By using the forced swimming test (FST), we investigated the effects of treatment with NAC on immobility subacutely in rats after oral dosing once a day for 2 days. Serum levels of cytokine interleukin-1 beta (IL-lbeta) and tumor necrosis factor-alpha (TNF-alpha) were determined by enzyme-linked immunosorbent assay (ELISA). Nuclear factor-2 (Nrf-2) and inducible nitric oxide synthases (iNOS) were analyzed by western blot method. NAC dose-dependently decreased immobility in the FST. NAC dosedependently decreased FST-induced increase of cytokine levels, as manifested by significantly stronger effects on IL-1 $\beta$ and TNF- $\alpha$ levels at higher doses than the lowest dose of NAC. Western blot analysis showed that Nrf-2 was significantly lower in the NAC-treated group than in the disease-induced group. The iNOS results were also significantly lower in the NAC-treated group than in the other groups. Considering FST results, the antidepressant effect of NAC is effective. Considering the results of cytokine and protein expression, this anti-depressant effect may be related to the anti-inflammatory effect. Therefore, it can be said that the anti-inflammatory effect of NAC increases the antidepressant effect in the FST experiment.
\end{abstract}

Key Words: Antidepressant agent, 2-Nonadecanone, FST, IL-1beta, TNF-alpha, iNOS, Nrf2

\section{INTRODUCTION}

Depression is a type of mood disorder characterized by hypochondriasis, decreased appetite, and insomnia. Depression is a disease that affects more than 100 million people worldwide (Broomhall et al., 2017). It is known that the nature of depression arises from physiological and anatomical problems. Antidepressants are divided into three categories, Tricyclic antidepressants (TCAs), selective serotonin reuptake inhibitors (SSRIs), and serotonin-norepinephrine reuptake inhibitors (SNRIs) (Agarwal et al., 2013). However, monoamine prescription, a typical depression treatment model, has limitations (Casey et al., 2017; Maglanoc et al., 2018), One of the causes of this is the result of adaptation failure to allostatic load (Benatti et al., 2016). When the stress is given, the human body activates the HPA axis and the sympathetic nerve (Juruena et al., 2014). When this situation prolongs, the inflammation reaction increases along with the depletion of the adrenal gland. The adaptation process to this stress is

Received: July 26, 2018 / Revised: August 31, 2018 / Accepted: August 31, 2018

* Professor.

${ }^{\dagger}$ Corresponding author: Kyung-Yae Hyun. Department of Clinical Laboratory Science, Dong-Eui University, Busan 47340, Korea.

Tel: +82-51-890-2683, Fax:+82-0505-182-6877, e-mail: kyhyun@deu.ac.kr

(C) The Korean Society for Biomedical Laboratory Sciences. All rights reserved.

(C) This is an Open Access article distributed under the terms of the Creative Commons Attribution Non-Commercial License (http://creativecommons.org/licenses/by-nc/3.0/) which permits unrestricted non-commercial use, distribution, and reproduction in any medium, provided the original work is properly cited. 
called allostasis and the extreme stress increases allostatic load (McEwen et al., 2000; Wilkinson et al., 2011). One of the goals of this experiment is to identify candidate agents that modulate inflammation mediated failure of adaptation to allostatic load in the treatment of depression.

The forced swimming test (FST), known as the desperate behavior assessment, is an experiment to evaluate the depressive effects of mice and rats (Bogdanova et al., 2013). Animal floating in water is in a state of extreme depression. FST test evaluates the depressed state of an animal by measuring floationg time. In this experiment, antidepressive effects of natural bioactive substances were evaluated through FST. Enzyme-linked immunosorbent assay (ELISA) of IL-1beta, TNF-alpha were performed to evaluate quantitatively the antiinflammatory effects of natural substances. For the analysis of inflammatory proteins, Nrf2 and iNOS were analyzed by western blotting method.

The antidepressant effect, antioxidative effect, and antidementia effect of Fomes fomentariushave been already reported in the previous studies (Zhang et al., 2015). However, there have been few studies on the 2-nonadecanone (NAC), the fatty acid component of Fomes fomentarius. This substance is expected to have an effective oxidizing effect and thus contributes to anti-inflammatory, antidepressant, and anti-dementia effects. This study was performed to examine NAC with respect to the antidepressant improvement over the anti-inflammatory ability of NAC.

\section{MATERIALS AND METHODS}

\section{Reagents}

All chemicals used in this study were of reagent grade, and most of them were purchased from Sigma (St. Louis, MO, USA). Reagents used in gel electrophoresis and western blot were purchased from Bio-Rad (Hercules, CA, USA). Nrf2 and iNOS antibodies were purchased from Santa Cruz Biotechnology Inc (SantaCruz, CA, USA) and Cell Signaling (Danvers, MA, USA). ELISA kit were purchased from Santa Cruz Biotechnology Inc (R\&D, USA).

\section{Experimental design}

Male Sprague-Dawley (SD) rats (aged at 4 weeks of weight

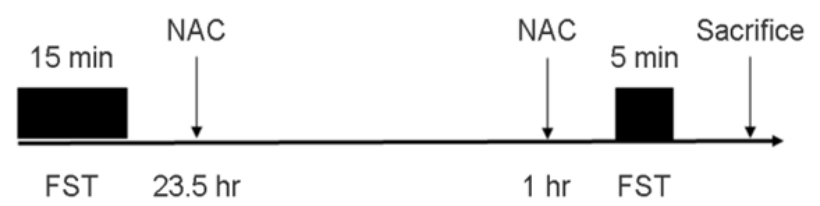

Fig. 1. Experimental design. Animals were sacrificed after the FST experiment, and blood, brain tissue, and soft tissues were collected as animal specimens.

range: 100 to $150 \mathrm{~g}$ ) were purchased from the Central Animal Inc. (Seoul, Korea) and housed cage in a light-controlled room (lights on from 9:00 AM to 9:00 PM) at a temperature of $20 \pm 2{ }^{\circ} \mathrm{C}$ and humidity of $60 \pm 5 \%$ with food and water available ad libitum. All experiments were approved by the ethics committee of Dong-Eui University (R2016-018) and were in accordance with the guidelines of the International Association for the Study of Pain (IASP). Total of $32 \mathrm{SD}$ rat were used, and the experimental schedule is shown in Fig. 1. After animal stabilization, the pre-FST (15 min) was performed. After that, NAC was administered intraperitoneally (i.p.) and NAC intraperitoneal administration was carried out again one hour before FST the next day. Animals were sacrificed after the FST experiment, and blood, brain tissue, and soft tissues were collected as animal specimens. Control group and experimental group were categorized as follows; Group I: Control group, saline intraperitoneal administration $(n=8)$, Group II: Vehicle, saline intraperitoneal administration, FST performed ( $\mathrm{n}=8)$, Group III: NAC1 mg/ $\mathrm{kg}$ intraperitoneal administration, FST performed $(\mathrm{n}=8)$, Group IV: NAC10 mg/kg intraperitoneal administration, FST performed $(n=8)$.

\section{Acute toxicity assay}

After NAC $250 \mathrm{mg} / \mathrm{kg}, 500 \mathrm{mg} / \mathrm{kg}$ and $1000 \mathrm{mg} / \mathrm{kg}$ intraperitoneally administering the test doses, the animals $(n=3)$ were kept under observation for 24 hours, for any behavioral effects and mortality. The numbers of survived and dead animals were noted by calculating the percentage of mortality.

\section{Forced swimming test}

A previous protocol reported for the FST (Yan et al., 2010) 
Table 1. Acute Toxicity test of NAC

\begin{tabular}{cccc}
\hline \hline Treatment & Dose $(\mathrm{mg} / \mathrm{kg})$ & No. of animals died $/ 3$ & $\%$ mortality \\
\hline & 250 & $0 / 3$ & 0 \\
NAC administered intraperitoneally & 500 & $0 / 3$ & 0 \\
& 1,000 & $0 / 3$ & 0 \\
\hline
\end{tabular}

was modified and followed. In brief, SD rat were placed into a cylinder (diameter $20 \mathrm{~cm}$ ) that was filled with $8 \mathrm{~L}$ of water (depth $30 \mathrm{~cm}, 22 \pm 3^{\circ} \mathrm{C}$ ). On the first day, SD rats were placed in the water for $15 \mathrm{~min}$ for habituation, dried in a heater, and returned to their home cage. On the second day, rats were placed in the water for $5 \mathrm{~min}$. The types of movement were classified into two types (swimming and climbing). The rats were considered immobile when they remained motionless and floated, and when they moved only to keep their heads above the water. The cylinder was washed, rinsed, and refilled with fresh water at $25 \pm 1{ }^{\circ} \mathrm{C}$ for every test.

\section{Enzyme immune assay of TNF-alpha and IL-1beta}

Serum level of TNF-alpha and IL-1beta were determined by an enzyme-linked immunesorbent assay (ELISA). Serum of the sacrificed animals was analyzed by three times dilution with commercial reagents. This analysis was used by commercial kits (BD Biosciences) according to the manufacturer's instructions.

\section{Western blot analysis of Nrf2 and iNOS}

SD rats of each group were euthanized by rapid decapitation after last behavioral experiments. Then brains were removed and hippocampus were rapidly dissected and frozen at $-80^{\circ} \mathrm{C}$ for protein study. Individual tissue samples were weighed and then homogenized in $500 \mu \mathrm{L}$ of ice-cold protein lysis buffer containing $20 \mathrm{mM}$ Tris- $\mathrm{HCl}(\mathrm{pH} 7.4), 1 \%$ Triton-X100, 1.5 mM EDTA, 40 mM KCl, 5\% glycerol, 0.5 $\mathrm{mM}$ dithiothreitol (DTT), $1 \mathrm{mM} \mathrm{NaF}, 1 \mathrm{mM}$ Phenylmethyl sulfonyl fluoride (PMSF) and proteinase inhibitor. The homogenates were centrifuged at $100,000 \times \mathrm{g}$ for $60 \mathrm{~min}$ at $4^{\circ} \mathrm{C}$. The supernatant was get from each sample, and an27 aliquot was taken to determine the total protein concentration using the Bradford Reagent. The proteins were then added sodium dodecylsulfate (SDS) loading buffers containing $0.1 \%$ of bromophenol blue and boiled for $5 \mathrm{~min}$. Then each sample were separated by SDS-polyacrylamide gel electrophoresis and transferred to a polyvinylidenefluoride (PVDF) membrane. The membrane was blocked with skim milk for $1 \mathrm{~h}$ and incubated with a primary antibody (Ab) against $\mathrm{Nrf} 2$ and iNOS, $\left(1: 1,000\right.$, Cell Signaling) for overnight at $4^{\circ} \mathrm{C}$, and then it was incubated with the HRP-conjugated secondary $\mathrm{Ab}$ (Cell Signaling) for $1 \mathrm{~h}$ at room temperature. The membranes were visualized using an ECL system and then developed on Hyperfilm (Amersham). The relative expression levels of all proteins were determined through a densitometry and normalized by actin.

\section{Data analysis}

Data are presented as the mean \pm standard error of the mean. ANOVA was used to compare the serial mean latency among groups in acquisition test of the Morris water maze. Fishe's least significant difference (LSD) test was used for post hoc comparisons after repeated measures ANOVA. Values with different letters are significantly different at $P$ $<0.05$, according to the Fisher's least significant difference (LSD) analysis. Statistical analyses were performed using a commercially available software package, SPSS version 12.0 (SPSS Inc., Chicago, IL). Graphs were drawn using Sigma Plot version 9.0 (Systat Software Inc., San Jose, CA).

\section{RESULTS}

\section{Acute toxicity study}

The acute toxicity of NAC was assessed in various doses $(250,500$, and $100 \mathrm{mg} / \mathrm{kg}$ ) (Table 1). During $24 \mathrm{hr}$ assessment of the important behavioral of toxicity, no mortality was observed at higher doses. 


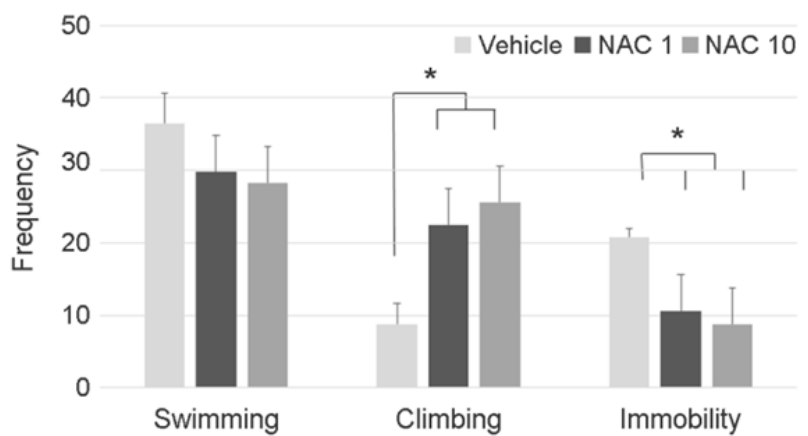

Fig. 2. Forced swimming test (FST). Prolonged swimming time and climbing time indicates decrease of fatigue. The data represent mean \pm standard error of mean of swimming, climbing and immobility ( $\mathrm{n}=8$ per group). *; $P<0.05$ vs. controls, as assessed by oneway ANOVA. Abbreviations: 2-Nonadecanone, NAC; 2-Nonadecanone $1 \mathrm{mg} / \mathrm{kg}$ i.p., NAC 1; 2-Nonadecanone $10 \mathrm{mg} / \mathrm{kg}$ i.p., NAC 10

\section{Forced swimming test}

The forced swimming test has recently been used to evaluate the anti-fatigue activities of certain agents (Cryan et al., 2005; Tan et al., 2012). Prolonged swimming time and climbing time indicates decrease of fatigue. According to Fig. 2, in the non-NAC group (vehicle), the inactivity time was significantly increased compared to the NAC group $(P$ $<0.05$ ). Swimming time showed no significant difference between the groups. However, climbing time was significantly different between the groups, and the results were expressed in a concentration dependent manner.

\section{Enzyme immune assay of TNF-alpha and IL-1beta}

The concentration of cytokine was measured using animal serum. Animals that did not give stress were set as control group. The results of inflammatory cytokines are shown in Fig. 3. The concentration of cytokines increased in all except the control group. In the FST - stimulated group without NAC administration (vehicle), a rapid increase in inflammatory cytokines was observed. Compared with the vehicle group (TNF-alpha, $218.5 \pm 12.1 \mathrm{ng} / \mathrm{mL}$; IL-1beta, $48.5 \pm$ $3.9 \mathrm{ng} / \mathrm{mL}$ ), there was a statistically significant difference between the NAC 1 (TNF-alpha, $113.8 \pm 7.4 \mathrm{ng} / \mathrm{mL}$; IL1beta, $25.1 \pm 2.1 \mathrm{ng} / \mathrm{mL}$ ) and NAC 10 (TNF-alpha, $98.7 \pm$ $8.1 \mathrm{ng} / \mathrm{mL}$; IL-1beta, $17.8 \pm 2.8 \mathrm{ng} / \mathrm{mL})$ groups $(P<0.05)$. Although not linear, the concentration of inflammatory cyto-

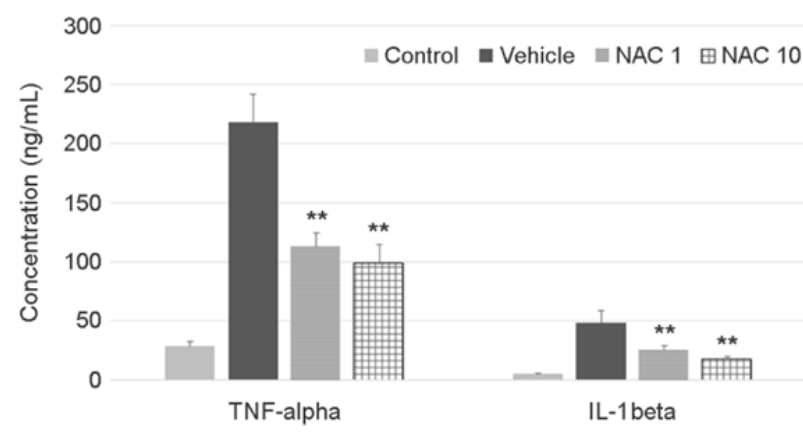

Fig. 3. Enzyme immune assay of TNF-alpha and IL-1beta. In the FST-stimulated group without NAC administration (vehicle), a rapid increase in inflammatory cytokines was observed. Compared with the vehicle group, there was a statistically significant difference between the NAC 1and NAC 10 groups ( ${ }^{*}, P<0.05$; **, $P<0.01$ ). The concentration of inflammatory cytokines in the NAC group was expressed in a concentration-dependent manner. Abbreviations: 2-Nonadecanone, NAC; 2-Nonadecanone $1 \mathrm{mg} / \mathrm{kg}$ i.p., NAC 1; 2 Nonadecanone $10 \mathrm{mg} / \mathrm{kg}$ i.p., NAC 10

kines in the NAC group was expressed in a concentrationdependent manner.

\section{Western blot analysis of Nrf2 and iNOS}

After animal sacrifice, specimens were collected around the limbic system where the corpus callosum was cut. Protein Nrf2 and iNOS analysis in brain tissue was measured and the results are shown in Fig. 4. Expression of other groups of proteins was expressed in multiples as compared to control group. Fig. 4 shows that the expression of iNOS was significantly different in the NAC $1(2.33 \pm 0.31)$ and NAC $(1.69 \pm 0.29)$ groups compared to the vehicle (3.79 $\pm 0.23)$ group. The protein expression of Nrf2 was significantly lower in the NAC group than in the vehicle group $(P$ $<0.05)$.

\section{DISCUSSION}

Inflammation is a kind of immune response caused by damage to tissues due to internal and external stimuli (Corn et al., 2015). The development of molecular biology has led to the discovery of various inflammatory markers and a new approach to inflammation. Today, inflammation is accepted as the basic pathology underlying almost all diseases. Mental illness is no exception, and disturbance of the immune system 
A

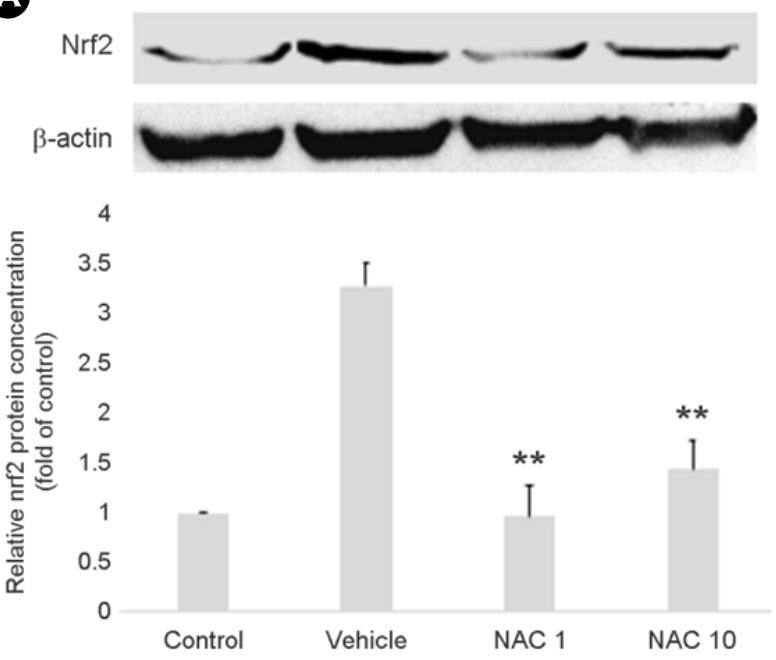

B

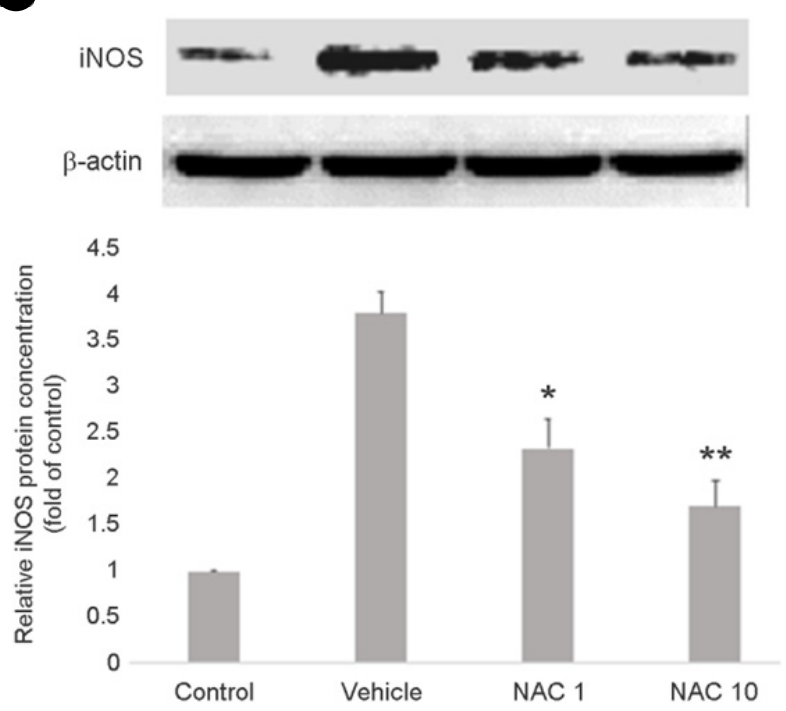

Fig. 4. Expression of protein in brain tissue. (A) Nrf2 protein expression (B) iNOS protein expression, Protein Nrf2 and iNOS analysis in brain tissue was measured and the results are shown in Fig. 4. Expression of other groups of proteins was expressed in multiples as compared to control group. Compared with the vehicle group, there was a statistically significant difference between the NAC 1and NAC 10 groups $(*, P<0.05 ; * *, P<0.01)$. Abbreviations: 2-Nonadecanone, NAC; 2-Nonadecanone $1 \mathrm{mg} / \mathrm{kg}$ i.p., NAC 1; 2-Nonadecanone $10 \mathrm{mg} / \mathrm{kg}$ i.p., NAC 10

and aggravation of the inflammatory response have become important factors in explaining the pathophysiology of mental disorders including depression (McGeer et al., 2006). The maintenance of homeostasis is the key to the health of the mind and body. Systems that control homeostasis, such as autonomic nervous system, immune system, and endocrine system, are web-like interaction around the brain. On the other hand, given stress, the human body activates the hypothalamic-pituitary-adrenal axis (HPA axis) and the sympathetic nerve to counteract it (Peila et al., 2006). If this situation persists for a long time, the sensitivity of body tissues to glu-cocorticoid is lowered, leading to so-called adrenal exhaustion and an increase in inflammatory response. This process of adapting to stress through physiological changes is called allostasis. Severe or repeated stress increases allostatic load resulting in failure of adaptation (Grande et al., 2012). Once changed, the failure tends not to return to the original state even after the stress is lost. In this article, it was hypothesized that depression of inflammation by NAC administration is effective for relieving depression.

Fig. 2 suggests that there is no doubt about the decline in animal depression due to NAC. However, unlike in other studies, we express immobility time and behavior time in frequency. The reason is that there are many subtle divisions between floating time and action time, and it is inappropriate to express it in time (sec). The immune system regulates the nervous system through a variety of cytokines secreted by immune cells. IL-1 and IL-2, which are representative inflammatory cytokines, increase the synthesis and circulation of serotonin, norepinephrine, and dopamine. At the same time, the activity of indolamine-2,3-dioxygenase (IDO), which degrades tryptophan, a precursor of serotonin, is increased (Schreck et al., 2010). This increase in IDO activity causes a decrease in serotonin. Recently, inflammatory markers with relatively consistent elevations in depression have been selected from two meta-analyzes: IL-6, TNF-alpha, TNF- $\beta 1$, IFN and $\mathrm{C}$-reactive protein. These inflammatory cytokines have the effect of increasing peripheral glucocorticoid resistance, inducing the depression of the HPA axis and lowering the utilization of serotonin by converting tryptophan to kynurenine rather than serotonin (Quagliato et al., 2018). From this viewpoint, the results of inflammatory cytokines in this study may be suspected to be related to depression. At Fig. 3, the expression of inflammatory cytokines in the depressed NAC group was decreased. Similar to the case of the depressed group, the expression level of inflammatory cytokines is also dependent on the NAC concentration. Although not 
firmly established, these results provide evidence that depressed depression by NAC administration is associated with a reduction in inflammatory cytokines due to the administration of the agent. The expression of the relevant protein was analyzed by a confirmation procedure for inflammation. Macrophages secrete various precursors in response to stress. In addition to inflammatory cytokines, macrophages express various inflammatory mediators such as nitric oxide (NO) and prostaglandin E2 (PGE2) through the expression of enzymes such as inducible nitric oxide synthase (iNOS) and cyclooxygenase-2 (COX-2) (Zhou et al., 2017). In this study, decreased expression of iNOS is another evidence of decreased inflammation in the brain (Fig. 4). In other words, it can be said that administration of NAC inhibited the expression of inflammation to stress in the body. Nuclear factor erythroid 2-related factor 2 (Nrf2) is known as the primary transcription factor for the cell defense phase corresponding to oxidative stress (Omar et al., 2017). The decrease in Nrf2 expression in the NAC-treated group could be attributed to the inhibition of inflammation in the brain by NAC administration (Fig. 4).

Recently, there has been research showing that treatment with anti-inflammatory drugs in combination with existing therapies is highly effective in depressed patients. There was a move to use IL-1 as a treatment for various neuropsychiatric disorders. In recent years, TNF-alpha has become a target for disease-modifying treatment of depression. These trends suggest that NAC used in this study may be a competitive antidepressant candidate drug.

\section{ACKNOWLEDGEMENTS}

This research was supported by the Korea Research Foundation (NRF-2017R1C1B5076959).

\section{CONFLICT OF INTEREST}

The authors declare no conflicts of interest.

\section{REFERENCES}

Agarwal P, Pan X, Sambamoorthi U. Depression treatment patterns among individuals with osteoarthritis: a cross sectional study. BMC Psychiatry. 2013. 22: 111-121.
Anne GB, Wendy J, Donald WH, Natasha ML. Upward counterfactual thinking and depression: A meta-analysis. Clinical Psychology. 2017. 55: 56-73.

Bogdanova OV, Kanekar S, D'Anci KE, Renshaw PF. Factors influencing behavior in the forced swim test. Physiol Behav. 2013. 13: 227-239.

Casey DA. Depression in Older Adults: A Treatable Medical Condition. Prim Care. 2017. 44: 499-510.

Corn JE, Vucic D. Ubiquitin in inflammation: the right linkage makes all the difference. Nat Struct Mol Biol. 2014. 21: 297 -300 .

Cryan JF, Valentino RJ, Lucki I. Assessing substrates underlying the behavioral effects of antidepressants using the modified rat forced swimming test. Neurosci Biobehav Rev. 2005. 29: 547-569.

Detke MJ, Lucki I. Detection of serotonergic and noradrenergic antidepressants in the rat forced swimming test: The effects of water depth. Behavioural Brain Research. 1996. 73: 43-46.

Grande I, Magalhães PV, Kunz M, Vieta E, Kapczinski F. Mediators of allostasis and systemic toxicity in bipolar disorder. Physiol Behav. 2012. 106: 46-50.

Maglanoc LA, Landrø NI, Jonassen R, Kaufmann T, CórdovaPalomera A, Hilland E, Westlye LT. Data-Driven Clustering Reveals a Link Between Symptoms and Functional Brain Connectivity in Depression. Biol Psychiatry Cogn Neurosci Neuroimaging. 2018. 6: 21-30.

McGeer PL, Rogers J, McGeer EG. Inflammation, anti-inflammatory agents and Alzheimer disease: the last 12 years. J Alzheimers Dis. 2006. 9: 271-276.

Omar NN, Tash RF. Fluoxetine coupled with zinc in a chronic mild stress model of depression: Providing a reservoir for optimum zinc signaling and neuronal remodeling. Pharmacol Biochem Behav. 2017. 160: 30-38.

Peila R, Launer LJ. Inflammation and dementia: epidemiologic evidence. Acta Neurol Scand Suppl. 2006. 185: 102-106.

Quagliato LA, Nardi AE. Cytokine alterations in panic disorder: A systematic review. J Affect Disord. 2018. 1: 91-96.

Schreck CB. Stress and fish reproduction: the roles of allostasis and hormesis. Gen Comp Endocrinol. 2010. 165: 549-556.

Tan W, Yu KQ, Liu YY, Ouyang MZ, Yan MH, Luo R, Zhao XS. Anti-fatigue activity of polysaccharides extract from Radix Rehmanniae Preparata. Int J Biol Macromol. 2012. 50: 59-62.

Yan HC, Cao X, Das M, Zhu XH, Gao TM. Behavioral animal models of depression. Neurosci Bull. 2010. 26: 327-337. 
Zhang. Chemical compositions and antiproliferation activities of the chloroform fraction from Pyropolyporus fomentarius in K562 cells. Hum Exp Toxicol. 2015. 34: 732-743.

Zhou XY, Zhang F, Ying CJ, Chen J, Chen L, Dong J, Shi Y, Tang M, Hu XT, Pan ZH, Xu NN, Zheng KY, Tang RX, Song YJ. Inhibition of iNOS alleviates cognitive deficits and depression in diabetic mice through downregulating the $\mathrm{NO} / \mathrm{sGC} / \mathrm{cGMP} /$
PKG signal pathway. Behav Brain Res. 2017. 30: 70-82.

https://doi.org/10.15616/BSL.2018.24.3.206

Cite this article as: Lee GH, Hyun KY. 2-Nonadecanone Alleviates Depression through Inflammation Relief in SD Rat. Biomedical Science Letters. 2018. 24: 206-212. 